\title{
Studies on the Preparation and Photocatalytic Performance of Bismuth Ferrite
}

\author{
Xinxin Wang ${ }^{1, a}$ and Yifa Dong ${ }^{2, b}$ \\ ${ }^{1}$ School of Chemistry and Bioengineering, Qilu Institute of Technology, Jinan, Shandong, 250200, China \\ ${ }^{2}$ School of Chemistry and Bioengineering, Qilu Institute of Technology, Jinan, Shandong, 250200, China
}

\begin{abstract}
Iron oxide and bismuth oxide powder are synthesized by the liquid phase precipitation method. Then a certain proportion of iron oxide, bismuth oxide and sodium chloride are mixed and ground to prepare the bismuth ferrite precursor by the molten salt method. The bismuth ferrite precursors are calcined at different temperatures to obtain bismuth ferrite photocatalyst. By studying the degradation performance of bismuth oxide, iron oxide and bismuth ferrite photocatalysts for methyl orange dyes, the best conditions for the degradation of methyl orange dyes by bismuth ferrite photocatalysts are found. The experimental results show that the bismuth ferrite prepared by calcining at $750^{\circ} \mathrm{C}$ has the best degradation. Compared with a single oxide, the photocatalytic performance of bismuth ferrite is better.
\end{abstract}

\section{Introduction}

As energy sources, coal and oil have brought convenience to mankind, but at the same time they have brought great damage to the earth's environment. Problems such as the greenhouse effect and air pollution are becoming more and more serious. Fossil energy is a non-renewable resource. With the development of advanced mining technology, fossil energy reserves have shown a state of exhaustion. We urgently need to break the traditional energy structure with fossil energy as the core, and look for cleaner and sufficient new energy $[1,2]$.

Among many new energy sources, the use of light energy is particularly prominent. In order to improve the efficiency of light energy conversion, the research work of light energy catalysis has been started [3-5]. In recent years, some multiferroic materials with larger refractive index and higher dielectric constant have been extensively studied in optoelectronic devices and infrared detection applications. Waste of resources and secondary pollution are avoided by the photocatalytic technology. Nanophotocatalytic technology is widely used in hot fields such as pollution control and self-cleaning materials. With the continuous deepening of research, bismuth ferrite nanomaterials have demonstrated their broad application prospects in the field of photocatalysis due to their obvious photocatalytic effects, high efficiency in degrading organics, and easy recycling [6-14].

Bismuth ferrite nanomaterials have excellent stability and high catalytic performance in photocatalysis. The preparation methods of bismuth ferrite nanomaterials mainly include sol-gel method [6-8], hydrothermal synthesis method [9-12], molten salt method [13, 14]. Bismuth ferrite powder with a particle size of $80-120 \mathrm{~nm}$ is synthesized by the sol-gel method [7]. Because the nanoparticles have a larger specific surface area, the contact area of the reaction is increased. As a result, its reactivity and reaction rate are improved, and the nanoparticles exhibit strong catalytic performance. Bismuth ferrite powders are synthesized by chemical coprecipitation method [10]. The results of photocatalysis experiments show that the degradation rate of bismuth ferrite photocatalyst to methyl orange solution could reach $92 \%$ under the conditions of ultraviolet light irradiation after $260 \mathrm{~min}$. It has high photocatalytic performance under ultraviolet light irradiation. Bismuth ferrite powders with different morphologies are successfully prepared by a controlled hydrothermal method [14].

By studying the degradation effect of bismuth ferrite powders of various shapes and appearances on Congo red dye, it is found that the smallest submicron cubic particles have the best photocatalytic effect on Congo red.

\section{Experimental}

Synthesis of Bismuth Ferrite Material. Bismuth oxide, iron oxide, and sodium chloride in a molar ratio are weighed respectively, and put them into a mortar for thorough mixing. A small amount of alcohol are added to grind. When the alcohol has evaporated, a small amount of alcohol are added again. The mixture are ground several times. The uniformly ground mixture are put into a drying oven at $60^{\circ} \mathrm{C}$ for $12 \mathrm{~h}$. Then the obtained powders are sintered at $650^{\circ} \mathrm{C}, 750^{\circ} \mathrm{C}$ and $850^{\circ} \mathrm{C}$ for $2 \mathrm{~h}$. The obtained products are washed with deionized water, and then put into a drying box for drying to obtain bismuth ferrite products, respectively.

Determination of the Maximum Absorption Wavelength of Methyl Orange. Methyl orange solution 
with a concentration of $10 \mathrm{mg} / \mathrm{L}$ is prepared. The absorbance of the test solution at different wavelengths is measured by the visible spectrophotometer, as shown in
Figure 1. It can be seen from the figure that the maximum wavelength of methyl orange is $460 \mathrm{~nm}$.

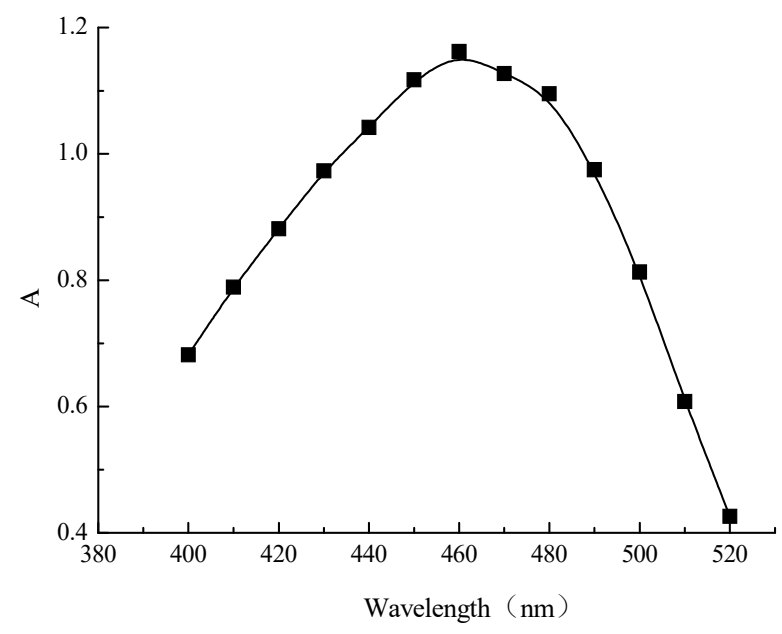

Figure 1. Absorption spectrum of methyl orange

\section{Results and discussion}

In this experiment, methyl orange solution is used as the test substrate to study the effect of different calcination temperatures and different catalysts on the degradation of methyl orange, and the analysis and discussion are carried out in combination with the characterization results. According to the relationship between absorbance and concentration, the degradation rate of the solution is calculated. The difference between the absorbance of the solution at the initial time and the measurement time and the percentage of the absorbance of the initial solution are used to define the degradation rate of the solution. The calculation formula of degradation rate is as follows:

$$
\mathrm{y}=\left[\left(\mathrm{A}_{0}-\mathrm{A}_{\mathrm{t}}\right) / \mathrm{A}_{0}\right] \times 100 \%
$$

In the formula, $\mathrm{A}_{0}$ represents the absorbance of the reference solution; $A_{t}$ represents the absorbance of the sample at $\mathrm{t}$ min during photodegradation; $\mathrm{y}$ represents the degradation rate of the substrate solution.

Drawing Standard Curve of Methyl Orange. Methyl orange solutions are prepared at concentrations of $1 \mathrm{mg} / \mathrm{L}, 5 \mathrm{mg} / \mathrm{L}, 10 \mathrm{mg} / \mathrm{L}, 15 \mathrm{mg} / \mathrm{L}$, and $20 \mathrm{mg} / \mathrm{L}$, respectively. And the absorbance is measured at $460 \mathrm{~nm}$. The standard curve of methyl orange is drawn, as shown in Figure 2.

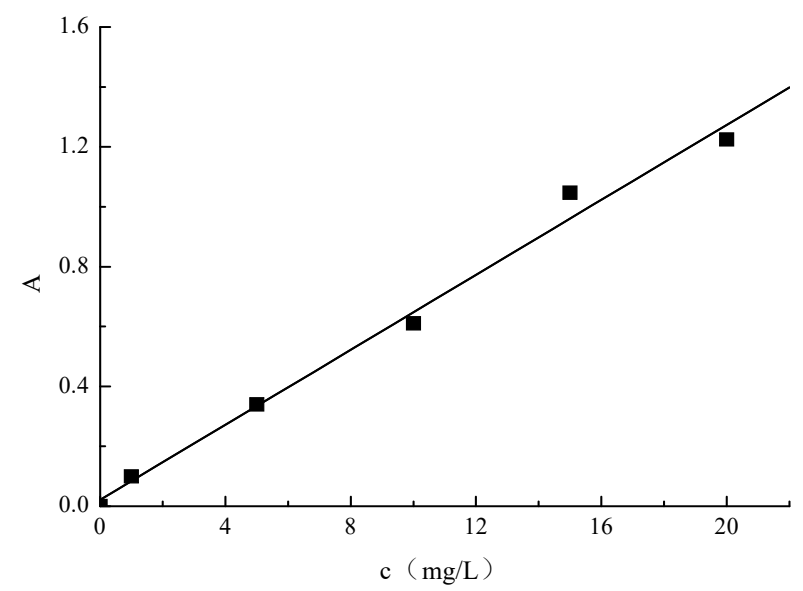

Figure 2. Standard curve of methyl orange

According to the measured data, a standard curve diagram of methyl orange is drawn. As can be seen from Figure 2, the linear equation of absorbance and concentration curve is:

$\mathrm{A}=0.00716+0.06971 \mathrm{c}$. Among them, $\mathrm{c}$ is the concentration of methylene blue solution, and its unit is $\mathrm{mg} / \mathrm{L}$.
Analysis of Calcination Temperature on Degradation Effect. The methyl orange solution with a concentration of $10 \mathrm{mg} / \mathrm{L}$ is put into three beakers, respectively. The prepared bismuth ferrite $\left(650^{\circ} \mathrm{C}\right)$, bismuth ferrite $\left(750^{\circ} \mathrm{C}\right)$, and bismuth ferrite $\left(850^{\circ} \mathrm{C}\right)$ are added into the above three solutions, respectively. The mixed liquids are placed in an ultrasonic cleaner for 
ultrasonic dispersion. Then, the mixed liquids are shaded and reacted on a multi-point magnetic stirrer for 30 minutes to reach the equilibrium of adsorption and desorption, and the initial absorbance is measured, respectively. The mixture are irradiated with a purple lamp for $2 \mathrm{~h}$ while stirring. During this period, samples are taken every 20 minutes. The samples are placed in a centrifuge for $5 \mathrm{~min}$. Using the deionized water as a reference, the absorbance is measured at $460 \mathrm{~nm}$ with a spectrophotometer. The degradation rate of the solution is calculated based on the absorbance, andthe drawing results are shown in Figure 3.

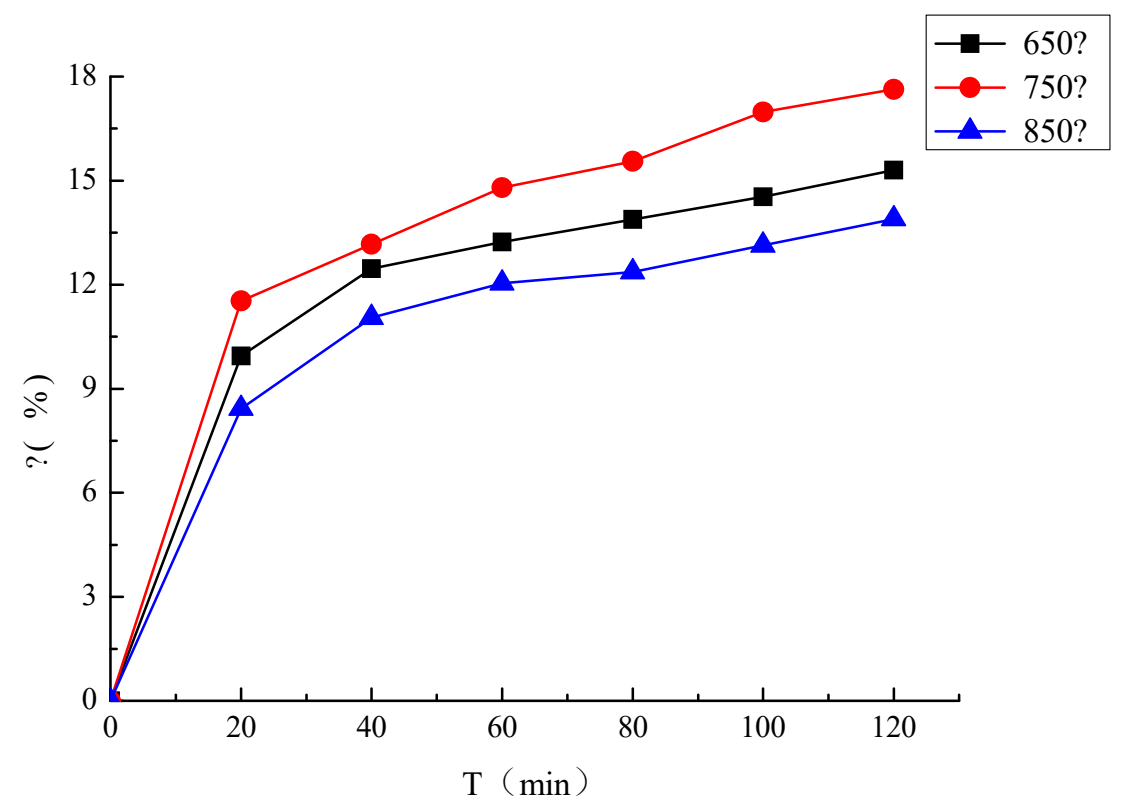

Figure 3. Effect of bismuth ferrite with different sintering temperature on the degradation rate of methyl orange

It can be seen from Figure 3 that the bismuth ferrite obtained at different sintering temperatures has different degradation effects on methyl orange. The bismuth ferrite obtained by sintering at $750^{\circ} \mathrm{C}$ has the best degradation effect, indicating that it has higher catalytic activity. When the calcination temperature is $650^{\circ} \mathrm{C}$, only a small amount of $\mathrm{BiFeO}_{3}$ phase exist in the sample. When the sintering temperature reaches $750^{\circ} \mathrm{C}$, the reaction is completed and a single-phase $\mathrm{BiFeO}_{3}$ powder is obtained. With the further increase of the sintering temperature, $\mathrm{BiFeO}_{3}$ begins to decompose, accompanied by the volatilization of $\mathrm{Bi}^{3+}$.
Comparison of Different Catalysts on Degradation Effects. The prepared iron oxide, bismuth oxide and bismuth ferrite $\left(750^{\circ} \mathrm{C}\right)$ are added into three methyl orange solutions, respectively. The mixed solutions are ultrasonically dispersed for $5 \mathrm{~min}$. Then, the mixed solutions are stirred for $30 \mathrm{~min}$ under light shielding, and the initial absorbance is measured, respectively. The mixed solutions are degraded under violet light irradiation for $2 \mathrm{~h}$ while stirring. The absorbance is measured every $20 \mathrm{~min}$. The relationship between the degradation rate of iron oxide, bismuth oxide, and bismuth ferrite $\left(750^{\circ} \mathrm{C}\right)$ to methyl orange and time is shown in Figure 4.

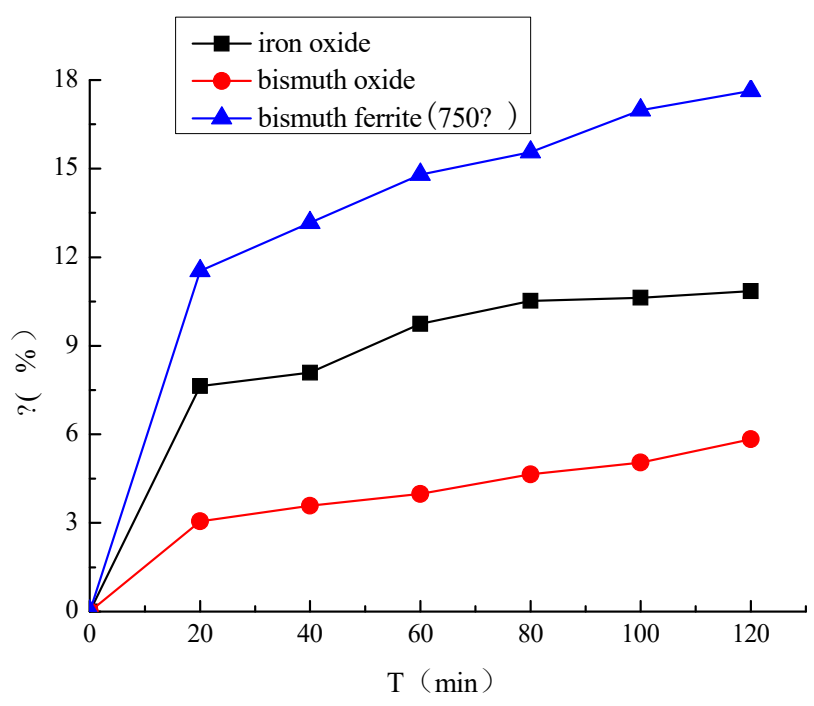

Figure 4. Effect of different catalysts on methyl orange degradation 
It can be seen from Figure 4 that the degradation rate of different catalysts gradually increases with the prolongation of the illumination time. Through comparison, it is found that the degradation effect of bismuth ferrite $\left(750^{\circ} \mathrm{C}\right)$ on methyl orange is better, and the degradation effect of iron oxide and bismuth oxide is slightly worse.

\section{Conclusion}

In this study, the catalytic performance of bismuth ferrite prepared at different calcination temperatures is tested, and it is concluded that the bismuth ferrite prepared by sintering at $750^{\circ} \mathrm{C}$ has the best performance. The photodegradation performance of different catalysts is tested, and it is found that the photocatalytic performance of bismuth ferrite is better than that of a single oxide. It can be seen that bismuth ferrite has better photocatalytic performance.

\section{Acknowledgments}

The authors are thankful to Research Project Foundation of Qilu Institute of Technology

for financial support of this work.

\section{References}

1. Byrne J A, Dunlop P S, Hamilton J W, e al. A review of heterogeneous photocatalysis for wate and surface disinfection[J]. Molecules, 2015, 20(4): 5574-5576.

2. Kudo A, Miseki Y. Heterogeneous photocatalyst materials for water splitting[J]. Chem. Soc. Rev., 2009, 38(1): 253-278.

3. Fujishima A, Honda K. Electrochemical photolysis of water at a semiconductor electrode[J]. Nature, 1972, 238(5358): 37-38.

4. Roland M. Semiconductor composites: strategies for enhancing charge carrier separation to improve photocatalytic activity[J]. Adv. Funct. Mater., 2014, 24: 2421-2440.

5. Fan W Q, Lai Q H, Zhang Q H, e al. Nanocomposites of $\mathrm{TiO}_{2}$ and reduced graphene oxide as efficient photocatalysts for hydrogen evolution[J]. J. Phys. Chem. C, 2011, 115: 10694-10701.

6. Zhang X, Lv J, Bourgeois L, et al. Formation and photocatalytic properties of bismuth ferrite submicrocrystals with tunable morphologies[J]. New Journal of Chemistry, 2011, 35(4): 937-941.

7. Gao F, Chen X Y, Yin K B, et al. Visible-light photocatalytic properties of weak magnetic $\mathrm{BiFeO}_{3}$ nanoparticles[J]. Adv. Mater., 2007, 19(19): 28892892.

8. Lin Z, Cai W, Jiang W, et al. Effects of annealing temperature on the microstructure, optical, ferroelectric and photovoltaic properties of $\mathrm{BiFeO}_{3}$ thin films prepared by sol-gel method[J]. Ceramics International, 2013, 39(8): 8729-8736.
9. Zheng Y Q, Tan G Q, Miao H Y, et al. Self-assembly growth of $\mathrm{BiFeO}_{3}$ powders prepared by microwavehydrothermal method[J]. Mater. Lett., 2011, 65(7): 1137-1140.

10. Liu Z K, Qi Y J, Lu C J. High efficient ultraviolet photocatalytic activity of $\mathrm{BiFeO}_{3}$ nanoparticles synthesized by a chemical coprecipitation process[J]. J. Mater. Sci: Mater Electron, 2010, 21(4): 380-384.

11. Fang Y L, Li Z Y, Xu S, et al. Optical properties and photocatalytic activities of spherical $\mathrm{ZnO}$ and flowerlike $\mathrm{ZnO}$ structures synthesized by facile hydrothermal method[J]. J. Alloy Compd., 2013, 575(20): 359-363.

12. Liu Y, Zuo R Z. Morphology and optical absorption of $\mathrm{Bi}_{2} \mathrm{Fe}_{4} \mathrm{O}_{9}$ crystals via mineralizer-assisted hydrothermal synthesis[J]. Particuology, 2013, 11: 581-587.

13. Chaudhuri A, Mandal K. Study of structural, ferromagnetic and ferroelectric properties of nanostructured barium doped bismuth ferrite $[\mathrm{J}]$. J. Magn. Mater., 2014, 353: 57-64.

14. Li S, Lin Y H, Zhang B P, Li J F, Nan C W. Controlled Fabrication of $\mathrm{BiFeO} 3$ uniform microcrystals and their magnetic and photocatalytic behaviors. J. Phys. Chem. C 2010, 114, 2903-2908. 